\title{
Existence of limit cycles in a tritrophic food chain model with Holling functional responses of type II and III
}

\author{
Gamaliel Bléa, Víctor Castellanos ${ }^{a *}$ and Jaume Llibre ${ }^{b}$
}

We are interested in the coexistence of three species forming a tritrophic food chain model. Considering a linear grow for the lowest trophic species, Holling III and Holling II functional response for the predator and the top-predator, respectively. We prove that this model has stable periodic orbits for adequate values of its parameters. Copyright (c) 2015 John Wiley \& Sons, Ltd.

Keywords: periodic orbit; Hopf bifurcation; population dynamics; Lyapunov coefficient

\section{Introduction}

The dynamic relationship between predators and their prey has long been studied and will continue being one of the dominant topics in both ecology and mathematical ecology due to its universality and importance, see for instance $[8,4]$. These problems may appear to be simple mathematically at first sight. In fact they are often very challenging and complicated.

Although the predator-prey theory has seen much progress in the last 40 years, there are still mathematical and ecological problems unsolved. In [3], the authors considered a model for three species that compete for three resources and they proved that the existence of two limit cycles evolves the coexistence equilibrium points. In [4] it is studied a model representing a tritrophic food chain composed of a logistic prey, a Holling type II predator and a Holling type II top-predator. They proved using the averaging theory the existence of a stable periodic orbit contained in the domain of interest. In [2] the authors analyzed a model representing a tritrophic food chain composed by a prey with linear grow and a functional response Holling type III for the predator and the top-predator. They proved using the averaging theory the existence of three-dimensional parameter families for which the model has two equilibrium points of zero-Hopf type contained in the domain of interest and also prove the existence of a simultaneously double zero-Hopf bifurcation.

In this paper we analyze a tritrophic food chain model considering Holling functional response of type III for the predator, Holling type II for the top-predator and linear grow for the prey.

a División Académica de Ciencias Básicas, UJAT, Km 1 Carretera Cunduacán-Jalpa de Méndez, C.p. 86690 Cunduacán Tabasco, México

b Departament de Matemátiques, Universitat Autónoma de Barcelona, 08193 Bellaterra, Barcelona, Catalonia, Spain

* Correspondence to: Víctor Castellanos. División Académica de Ciencias Básicas, UJAT, Km 1 Carretera Cunduacán-Jalpa de Méndez, c.p. 86690 Cunduacán Tabasco, México. E-mail; vicasujat.mx 
A tritrophic food chain model with functional response $f(x)$ and $g(y)$ for the middle and the highest trophic species, respectively, has the form

$$
\begin{aligned}
\dot{x} & =\rho x-f(x) y, \\
\dot{y} & =c y f(x)-g(y) z-d y, \\
\dot{z} & =z\left(e g(y)-d_{2}\right),
\end{aligned}
$$

where $x$ represents the prey, that gets eaten by a species of density $y$ (mesopredator) that feeds the species with density $z$ (super-predator). The parameters $\rho, c, d, d_{2}$ and $e$ are positive constants.

The functional responses of the mesopredator $f(x)$, and the super-predator $g(x)$ satisfy

$$
f(0)=g(0)=0, \quad \frac{d f(x)}{d x} \geq 0 \text { and } \frac{d g(y)}{d y} \geq 0 \quad \forall x, y \geq 0
$$

There are many functions that satisfy the above conditions, for example the functional responses of predation include the usual functions found in the literature (see, e.g., [5]). In this paper we will consider that $f$ is Holling type III and $g$ is Holling type II, more precisely

$$
f(x)=\frac{a_{1} x^{2}}{x^{2}+b_{1}} \text { and } g(y)=\frac{a_{2} y}{y+b_{2}}
$$

where $a_{1}, a_{2}, b_{1}$ and $b_{2}$ are positive constants.

Consequently the tritrophic food chain model that we will study is

$$
\begin{aligned}
& \dot{x}=x\left(\rho-\frac{a_{1} x y}{x^{2}+b_{1}}\right), \\
& \dot{y}=y\left(c \frac{a_{1} x^{2}}{x^{2}+b_{1}}-\frac{a_{2} z}{y+b_{2}}-d\right), \\
& \dot{z}=z\left(e \frac{a_{2} y}{y+b_{2}}-d_{2}\right) .
\end{aligned}
$$

For ecological considerations the domain of interest $\Omega$ is the positive octant of $\mathbb{R}^{3}$, i.e. $\Omega$ is the region $x>0, y>0$ and $z>0$.

We give necessary and sufficient conditions on the parameters to guarantee the existence of equilibrium points of the differential system (2) in the region of interest $\Omega$. Our main result shows that system (2) can exhibit Hopf bifurcations in one of their equilibria, and for any convenient values of the parameters the Hopf periodic orbit is stable.

To simply the expressions thought the paper we define

$$
k^{2}=a_{1}^{2} b_{2}^{2} d_{2}^{2}-4 b_{1}\left(d_{2}-a_{2} e\right)^{2} \rho^{2}, \quad k>0
$$

Solving this equation (3) for $b_{1}$, we write $b_{1}$ in terms of $k$

$$
b_{1}=\frac{a_{1}^{2} b_{2}^{2} d_{2}^{2}-k^{2}}{4\left(d_{2}-a_{2} e\right)^{2} \rho^{2}}
$$

Also we do

$$
\begin{aligned}
& B_{1}=d_{2}-a_{2} e, \\
& B_{2}=a_{1} b_{2} d_{2}-k, \\
& B_{3}=a_{1} b_{2} c d_{2}-2 b_{2} d d_{2}-c k .
\end{aligned}
$$

Theorem 1 If the parameters involved in system (2) satisfy the hypothesis
H1 $a_{2} e-d_{2}>0$,
H2 $a_{1} b_{2} d_{2}-k>0$,
H3 $a_{1}^{2} b_{2}^{2} d_{2}^{2}-4 b_{1}\left(d_{2}-a_{2} e\right)^{2} \rho^{2} \geq 0$,
H4 $a_{1} b_{2} c d_{2}-2 b_{2} d d_{2}-c k>0$ 
then we have an equilibrium point $p_{1}$ in the positive octant of $\mathbb{R}^{3}$. If additionally the hypothesis

H5 $-a_{2}^{2} b_{2}^{2} e^{2} k B_{2}^{2}\left(a_{1} a_{2} b_{2} d_{2} e-B_{1} k\right)+4 d_{2} B_{1}^{6} \rho^{2} \omega^{2}>0$,

is satisfied and the first Lyapunov coefficient $\ell\left(p_{1}\right)$ (see Appendix) at $p_{1}$ is not zero, then we have a Hopf bifurcation in $p_{1}$.

Theorem 2 If the parameters involved in system (2) satisfy the hypothesis H1, H3 and

H4' $a_{1} b_{2} c d_{2}-2 b_{2} d d_{2}+c k>0$,

then we have an equilibrium point $p_{2}$ in the positive octant of $\mathbb{R}^{3}$. But the standard sufficient conditions which guarantee the existence of a Hopf bifurcation in $p_{2}$ do not hold.

\section{Equilibrium points in the positive octant of $\mathbb{R}^{3}$}

Doing a rescaling of the time, we get that the differential system (2) is equivalent to the following differential system,

$$
\begin{aligned}
& \dot{x}=x\left(b_{2}+y\right)\left(-a_{1} x y+b_{1} \rho+x^{2} \rho\right), \\
& \dot{y}=-y\left(b_{1} b_{2} d-a_{1} b_{2} c x^{2}+b_{2} d x^{2}+b_{1} d y-a_{1} c x^{2} y+d x^{2} y+a_{2} b_{1} z+a_{2} x^{2} z\right), \\
& \dot{z}=-\left(b_{1}+x^{2}\right)\left(b_{2} d_{2}+d_{2} y-a_{2} e y\right) z .
\end{aligned}
$$

The equilibrium points of differential systems (5) in the region of interest $\Omega$, are the solution of the equations

$$
\begin{aligned}
-a_{1} x y+b_{1} \rho+x^{2} \rho & =0, \\
b_{1} b_{2} d-a_{1} b_{2} c x^{2}+b_{2} d x^{2}+b_{1} d y-a_{1} c x^{2} y+d x^{2} y+a_{2} b_{1} z+a_{2} x^{2} z & =0 \\
b_{2} d_{2}+d_{2} y-a_{2} e y & =0
\end{aligned}
$$

whose solutions are

$$
\begin{aligned}
& p_{1}=\left(\frac{a_{1} b_{2} d_{2}-k}{2\left(a_{2} e-d_{2}\right) \rho}, \frac{b_{2} d_{2}}{a_{2} e-d_{2}}, \frac{e\left(a_{1} b_{2} c d_{2}-2 b_{2} d d_{2}-c k\right)}{2 d_{2}\left(a_{2} e-d_{2}\right)}\right), \\
& p_{2}=\left(\frac{a_{1} b_{2} d_{2}+k}{2\left(a_{2} e-d_{2}\right) \rho}, \frac{b_{2} d_{2}}{a_{2} e-d_{2}}, \frac{e\left(a_{1} b_{2} c d_{2}-2 b_{2} d d_{2}+c k\right)}{2 d_{2}\left(a_{2} e-d_{2}\right)}\right) .
\end{aligned}
$$

So the following result guarantee the existence of two non trivial equilibrium points in $\Omega$.

Lemma 3 The equilibrium point $p_{1}$ of differential system (5) is in the positive octant of $\mathbb{R}^{3}$ if and only if the parameters satisfy the conditions $\mathbf{H 1}, \mathbf{H} 2, \mathbf{H} 3$ and $\mathbf{H} 4$. The equillibrium point $p_{2}$ of the differential system (5) is in the positive octant of $\mathbb{R}^{3}$ if and only if the parameters satisfy the conditions $\mathbf{H 1}, \mathbf{H} \mathbf{2}$ and $\mathbf{H} \mathbf{4}$

Proof. The proof is a direct consequence of the expression of $p_{1}$ and $p_{2}$.

We note that always that $p_{1}$ is in the positive octant of $\mathbb{R}^{3}$ also $p_{2}$ is there.

Now our goal is to determine when the equilibrium points $p_{1}$ and $p_{2}$ exhibits a Hopf bifurcation. In this sense we show the existence of parameters where the equilibrium points have a pair of pure imaginary eigenvalues and a nonzero real eigenvalue and using a Kuznetsov's Theorem we shall prove the existence of a Hopf bifurcation. The tool provided by the Kuznetsov Theorem is a good theory for studying the Hopf bifurcation, in particular in [1] it is applied in the repressilator equation of dimension six for proving the existence of a supercritical Hopf bifurcation. 


\section{First Lyapunov coefficient and Hopf bifurcation}

\subsection{Kuznetsov theorem}

When a differential system

$$
\dot{x}=F(x, \mu),
$$

in $\mathbb{R}^{n}$ has an equilibrium point $p$ having eigenvalues $\pm \omega i$, it is a candidate to exhibit a Hopf bifurcation, that is a local bifurcation in which an equilibrium point of a differential system loses stability as a pair of complex conjugate eigenvalues of the linearization, around the equilibrium point, cross the imaginary axis of the complex plane.

In order to show that this bifurcation takes place it is necessary to compute the first Lyapunov coefficient $\ell_{1}(p)$ of the differential system at the equilibrium $p$. When $\ell_{1}(p)<0$ the point $p$ is a weak focus of the differential system restricted to the central surface of $p$, associated to the pair of complex eigenvalues which cross the imaginary axis, and the limit cycle that emerges from $p$ is stable. In this case the Hopf bifurcation is called supercritical. When $\ell_{1}\left(p_{1}\right)>0$ the point $p$ is also a weak focus of the differential system restricted to the central surface of $p$ but the limit cycle that born from $p$ is unstable. In this second case we say that the Hopf bifurcation is subcritical. For more details on the Hopf bifurcation see for instance the book of Kuznetsov [7].

In this work we use the following result presented on page 180 of the book [7] for computing the first Lyapunov constant $\ell_{1}\left(p_{1}\right)$ at the equilibrium $p_{1}$.

Theorem 4 (Kuznetsov, 2004) Let $\dot{x}=F(x)$ be a differential system having $p_{0}$ as an equilibrium point. Consider the third order Taylor approximation of $F$ around $p_{0}$ given by

$$
F(x)=A x+\frac{1}{2 !} B(x, x)+\frac{1}{3 !} C(x, x, x)+O\left(|x|^{4}\right) .
$$

Assume that $A$ has a pair of purely imaginary eigenvalues $\pm \omega i$. Let $q$ be the eigenvector of $A$ corresponding to the eigenvalue wi, normalized so that $q \bar{q}=1$, where $\bar{q}$ is the conjugate vector of $q$. Let $p$ be the adjoint eigenvector such that $A^{T} p=-$ wip and $\bar{p} \cdot q=1$. If I denotes the identity matrix, then the first Liapunov constant $I_{1}\left(p_{0}\right)$ of the system $\dot{x}=F(x)$ at the equilibrium point $p_{0}$ is

$$
\frac{1}{2 \omega} \operatorname{Re}\left(\bar{p} \cdot C(q, q, \bar{q})-2 \bar{p} \cdot B\left(q, A^{-1} B(q, \bar{q})\right)+\bar{p} \cdot B\left(\bar{q},(2 \omega i l-A)^{-1} B(q, q)\right)\right)
$$

\subsection{Hopf bifurcation at equilibrium point $p_{1}$}

In the next two results we characterize when the equilibrium points $p_{1}$ and $p_{2}$ have complex eigenvalues with real part zero. Hence system (5) will be a candidate to exhibit a Hopf bifurcation.

Proposition 5 If the parameters satisfy the hypothesis $\mathrm{H1}_{1} \mathrm{H}_{2}, \mathrm{H} 3, \mathrm{H} 4$ and $\mathrm{H} 5$, then there exists a parameter set such that the equilibrium points $p_{1}$ is in the positive octant of $\mathbb{R}^{3}$ and have a pair of pure imaginary eigenvalues and the other eigenvalue is a non zero real.

Proof. The characteristic polynomial of the linear approximation of the tritropic system (5) at the equilibrium point $p_{1}$ is

$$
p(\lambda)=-\lambda^{3}+A_{2} \lambda^{2}+A_{1} \lambda+A_{0}
$$

where,

$$
\begin{aligned}
& A_{0}=-\frac{a_{1}^{2} a_{2}^{2} b_{2}^{4} d_{2}^{2} e^{2} B_{2}^{3} k B_{3}}{\left(16 B_{1}^{8} \rho^{5}\right.} \\
& A_{1}=\frac{a_{1} a_{2} b_{2}^{2} e B_{2}^{2}\left(a_{1} b_{2} d_{2}^{2} B_{1} B_{3}+\left(-a_{1}^{2} a_{2} b_{2}^{2} c d_{2}^{2} e+b_{2}\left(a_{1} c-2 d\right) d_{2}^{2} k-c B_{1} k^{2}\right) \rho\right)}{8 B_{1}^{6} \rho^{4}} \\
& A_{2}=\frac{b_{2} B_{2}\left(a_{1} d_{2} B_{3}-2 a_{2} e k \rho\right)}{4 B_{1}^{3} \rho^{2}}
\end{aligned}
$$


Now imposing the condition that $p(\lambda)=-(\lambda-\alpha)(\lambda+\omega i)(\lambda-\omega i)$, we obtain a system of three polynomial equations, that correspond to the coefficients of the terms of degree 0,1 and 2 in $\lambda$. Since the variables $c, d$ and $\alpha$ are of degree one in this system, then we have a linear system in this variables whose solution is

$$
\begin{aligned}
& c=-\frac{B_{1}^{5} \rho^{4} \omega^{2}\left(a_{2}^{2} b_{2}^{2} e^{2} k^{2} B_{2}^{2}+4 B_{1}^{6} \rho^{2} \omega^{2}\right)}{a_{1} a_{2}^{2} b_{2}^{2} e^{2} B_{2}^{3}\left(a_{1} b_{2} d_{2}+k\right)\left(a_{1} a_{2}^{2} b_{2}^{3} d_{2} e^{2} k B_{2}^{2}-4 B_{1}^{5} \rho^{3} \omega^{2}\right)}, \\
& d=-\frac{4 B_{1}^{5} \rho^{4} \omega^{2}\left(-a_{2}^{2} b_{2}^{2} e^{2} k B_{2}^{2}\left(a_{1} a_{2} b_{2} d_{2} e-B_{1} k\right)+4 d_{2} B_{1}^{6} \rho^{2} \omega^{2}\right)}{a_{1} a_{2}^{2} b_{2}^{3} d_{2}^{2} e^{2} B_{2}^{2}\left(a_{1} b_{2} d_{2}+k\right)\left(a_{1} a_{2}^{2} b_{2}^{3} d_{2} e^{2} k B_{2}^{2}-4 B_{1}^{5} \rho^{3} \omega^{2}\right)}, \\
& \alpha=-\frac{a_{1} a_{2}^{3} b_{2}^{4} d_{2} e^{3} B_{2}^{3} k^{2}}{2 a_{1} a_{2}^{2} b_{2}^{3} d_{2} e^{2}\left(-d_{2}+a_{2} e\right)^{3} k B_{2}^{2} \rho+8 B_{1}^{8} \rho^{4} \omega^{2}} .
\end{aligned}
$$

From the hypotheses of the proposition, we have that $B_{1}<0, B_{2}>0$ and $B_{3}>0$. Thus $c$ is positive. On the other hand, the denominator of $d$ is positive, so, we only verify the sign of numerator to know the sign of $d$. As $B_{1}<0$ then $B_{1}^{5}<0$, and so $-4 B_{1}^{5} \rho^{4} \omega^{2}>0$ in the expression of $d$, therefore the sign of $d$ is determined by the sign of the expression in the parenthesis of numerator,

$$
-a_{2}^{2} b_{2}^{2} e^{2} k B_{2}^{2}\left(a_{1} a_{2} b_{2} d_{2} e-B_{1} k\right)+4 d_{2} B_{1}^{6} \rho^{2} \omega^{2}
$$

that is positive by hypothesis $\mathbf{H 5}$. Hypothesis $\mathbf{H} \mathbf{5}$ is realizable because this is a polynomial equation of degree two in the variable $\omega$ of the form $t_{0}+t_{1} \omega^{2}$ with $t_{0}<0$ and $t_{1}>0$.

With these values of the parameters the equilibrium point $p_{1}$ takes the form

$$
p_{1}=\left(-\frac{B_{2}}{2 B_{1} \rho},-\frac{b_{2} d_{2}}{B_{1}}, \frac{4 a_{2} e^{2} B_{1}^{4} k \rho^{4} \omega^{2}}{a_{1} d_{2}^{2}\left(a_{1} a_{2}^{2} b_{2}^{3} d_{2} e^{2} k B_{2}^{2}-4 B_{1}^{5} \rho^{3} \omega^{2}\right)}\right),
$$

and the linear approximation at this point has a pair of pure imaginary eigenvalues $\omega i,-\omega i$ and one real, $\alpha$. It is clear that $p_{1}$ is in the positive octant.

\section{Proof of main results}

\subsection{Proof of Theorem 1}

Lemma 3 guarantees the existence of parameter families for which the equilibrium points $p_{1}$ and $p_{2}$ are in the positive octant of $\mathbb{R}^{3}$ and Proposition 5 establishes that also there is a family of parameters for which the equilibrium point $p_{1}$ has a pair of pure imaginary eigenvalues and one real eigenvalues. Theorem 1 is our main result, which guarantees the existence of Hopf bifurcation at the point $p_{1}$ and for convenient values of the parameters it will be stable. Example 6 shows that these last claims are satisfied for some values of the parameters.

In order that this bifurcation takes place we compute the first Lyapunov coefficient $\ell_{1}\left(p_{1}\right)$ of the differential system at the equilibrium $p_{1}$. This calculation is done using the formula of the Kuznetsov's Theorem.

Let $p_{1}$ be as in (8) and consider a linear change of variable to translate $p_{1}$ to at the origin of coordinates, after that change we obtain a differential system

$$
\dot{X}=F(X)
$$

with $X=(x, y, z)$ and $F(0)=0$. Denote the vector field

$$
F(x, y, z)=\left(f_{1}(x, y, z), f_{1}(x, y, z), f_{1}(x, y, z)\right)
$$

associated to this differential system. Then we compute the linear part $A$, the bilinear $B$ and trilinear $C$ forms of the Taylor expanssion of the function $F$. 
The linear part of system (9) at 0 is

$$
A=\left(\begin{array}{ccc}
\frac{a_{2} b_{2} e B_{2} k}{2 B_{1}^{3} \rho} & \frac{a_{1} a_{2} b_{2} e b_{2}^{2}}{4 B_{1}^{3} \rho^{2}} & 0 \\
\frac{4 B_{1}^{2} \rho^{3} \omega^{2}\left(a_{2}^{2} b_{2}^{2} e^{2} k^{2} b_{2}^{2}+4 B_{1}^{6} \rho^{2} \omega^{2}\right)}{a_{1} a_{2} b_{2} e B_{2}^{2} B_{4}} & \frac{2 a_{2} b_{2} e B_{1}^{2} B_{2} k \rho^{2} \omega^{2}}{B_{4}} & \frac{a_{1} a_{2} b_{2}^{2} d_{2}^{2} B_{2}}{2 B_{1}^{3} \rho^{2}} \\
0 & \frac{2 a_{2} b_{2} e^{2} B_{1}^{3} B_{2} k \rho^{2} \omega^{2}}{-d_{2} B_{4}} & 0
\end{array}\right),
$$

where

$$
B_{4}=a_{1} a_{2}^{2} b_{2}^{3} d_{2} e^{2} k B_{2}^{2}-4 B_{1}^{5} \rho^{3} \omega^{2} .
$$

The eigenvalues of $A$ are

$$
-i \omega, \quad i \omega, \quad-\frac{a_{1} a_{2}^{3} b_{2}^{4} d_{2} e^{3} B_{2}^{3} k^{2}}{8 B_{1}^{8} \rho^{4} \omega^{2}-2 a_{1} a_{2}^{2} b_{2}^{3} d_{2} e^{2} B_{1}^{3} k B_{2} 2 \rho} .
$$

The eigenvector $q=\left(q_{11}, q_{12}, q_{13}\right)$ of $A$ corresponding to eigenvalue $i \omega$ normalized such that $q \cdot \bar{q}=1$, where $\bar{q}$ is the conjugate vector of $q$ is presented in the Appendix. The normalized adjoint eigenvector $p=\left(p_{11}, p_{12}, p_{13}\right)$ of transpose matrix $A$ corresponding to the eigenvalue $-i \omega$, with the property that $\bar{p} \cdot q=1$ is also in the Appendix.

The calculus of the bilinear $B$ and trilinar $C$ forms, are provided in the Appendix because they have large expressions. So we have all the elements to compute the first Lyapunov coefficient $\ell\left(p_{1}\right)$ at $p_{1}$. The expression of $\ell\left(p_{1}\right)$ is given in the Appendix. This conclude, the proof of the theorem.

\subsection{Proof of Theorem 2}

To proof this theorem we do an equivalent procedure as the one done in the proof of Theorem 1 . From the expression of $p_{2}$ establish in (6) we have that it is in the positive octant of $\mathbb{R}^{3}$. The characteristic polynomial of the linear approximation of system (5) at the equilibrium $p_{2}$ is

$$
p(\lambda)=-\lambda^{3}+C_{2} \lambda^{2}+C_{1} \lambda+C_{0}
$$

where,

$$
\begin{aligned}
& C_{0}=-\frac{a_{1}^{2} a_{2}^{2} b_{2}^{4} d_{2}^{2} e^{2} k D_{1}^{3} D_{3}}{16 B_{1}^{8} \rho^{5}}, \\
& C_{1}=\frac{a_{1} a_{2} b_{2}^{2} e D_{1}^{2}\left(-a_{1} b_{2} d_{2}^{2} B_{1} D_{2}+\left(a_{1}^{2} a_{2} b_{2}^{2} c d_{2}^{2} e+b_{2}\left(a_{1} c-2 d\right) d_{2}^{2} k+c B_{1} k^{2}\right) \rho\right)}{8 B_{1}^{6} \rho^{4}}, \\
& C_{2}=\frac{b_{2} D_{1}\left(a_{1} d_{2} D_{2}+2 a_{2} e k \rho\right)}{4 B_{1}^{3} \rho^{2}}, \\
& D_{1}=a_{1} b_{2} d_{2}+k, \\
& D_{2}=a_{1} b_{2} c d_{2}-2 b_{2} d d_{2}+c k .
\end{aligned}
$$

Similarly imposing the condition that $p(\lambda)=-(\lambda-\alpha)(\lambda+\omega /)(\lambda-\omega /)$, we obtain a system of three polynomial equations, that are linear in the parameters $c, d$ and $\alpha$. The solution of this system in terms of these variables is

$$
\begin{aligned}
& c=\frac{8 B_{1}^{5} \rho^{4} \omega^{2}\left(a_{2}^{2} b_{2}^{2} e^{2} k^{2}\left(a_{1} b_{2} d_{2}+k\right)^{2}+4 B_{1}^{6} \rho^{2} \omega^{2}\right)}{a_{1} a_{2}^{2} b_{2}^{2} e^{2} B_{2} D_{1}^{3}\left(a_{1} a_{2}^{2} b_{2}^{3} d_{2} e^{2} k D_{1}^{2}+4 B_{1}^{5} \rho^{3} \omega^{2}\right)}, \\
& d=\frac{4 B_{1}^{5} \rho^{4} \omega^{2}\left(a_{2}^{2} b_{2}^{2} e^{2} k D_{1}^{2}\left(a_{1} a_{2} b_{2} d_{2} e+B_{1} k\right)+4 d_{2} B_{1}^{6} \rho^{2} \omega^{2}\right)}{a_{1} a_{2}^{2} b_{2}^{3} d_{2}^{2} e^{2} B_{2} D_{1}^{2}\left(a_{1} a_{2}^{2} b_{2}^{3} d_{2} e^{2} k D_{1}^{2}+4 B_{1}^{5} \rho^{3} \omega^{2}\right)}, \\
& \alpha=-\frac{a_{1} a_{2}^{3} b_{2}^{4} d_{2} e^{3} k^{2} D_{1}^{3}}{2 a_{1} a_{2}^{2} b_{2}^{3} d_{2} e^{2} B_{1}^{3} k D_{1}^{2} \rho+8 B_{1}^{8} \rho^{4} \omega^{2}} .
\end{aligned}
$$

The equilibrium point $p_{2}$ with the values of parameters given in (10) takes the form

$$
p_{2}=\left(-\frac{D_{1}}{2 B_{1} \rho},-\frac{b_{2} d_{2}}{B_{1}}, \frac{4 a_{2} e^{2} B_{1}^{4} k \rho^{4} \omega^{2}}{a_{1} d_{2}^{2}\left(a_{1} a_{2}^{2} b_{2}^{3} d_{2} e^{2} k D_{1}^{2}+4 B_{1}^{5} \rho^{3} \omega^{2}\right)}\right) .
$$


The linear approximation at this point has a pair of pure imaginary eigenvalues $\omega i,-\omega i$ and one real, $\alpha$ as we need for apply the Kuznetsov Theorem. With these values of the parameters $c$ and $d$, the equillibrium point $p_{2}$ remains in the positive octant of $\mathbb{R}^{3}$ if the condition

$$
a_{1} a_{2}^{2} b_{2}^{3} d_{2} e^{2} k D_{1}^{2}+4 B_{1}^{5} \rho^{3} \omega^{2}>0
$$

is satisfied. But in this case the parameter $c$ is negative and this is not possible because in our model all parameter are positive. This complete the proof of the theorem.

\subsection{Examples}

Theorem 1 is our main result, which guarantees the existence of Hopf bifurcation at the point $p_{1}$. Example 6 shows that this theorem is not empty.

Example 6 We show a concrete example where Theorem 1 holds and where the Hopf periodic orbit is stable. Consider the parameters values $a_{1}=1, a_{2}=2, b_{2}=2, d_{2}=1, e=2, k=1$. Then, from (4) and (7) we have

$$
\begin{aligned}
b_{1} & =\frac{1}{12 \rho^{2}}, \\
c & =\frac{81 \rho^{4} \omega^{2}\left(64+2916 \rho^{2} \omega^{2}\right)}{8\left(128+972 \rho^{3} \omega^{2}\right)}, \\
d & =\frac{81 \rho^{4} \omega^{2}\left(-704+2916 \rho^{2} \omega^{2}\right)}{32\left(128+972 \rho^{3} \omega^{2}\right)}, \\
\alpha & =-\frac{1024}{6912 \rho+52488 \rho^{4} \omega^{2}} .
\end{aligned}
$$

Note that $b_{1}$ and $c$ are always positive reals numbers and if

$$
\rho \omega>\frac{4}{27} \sqrt{11}
$$

then $d$ is positive too. The eigenvalue $\alpha$ is always negative.

All the hypothesis of Theorem 1 hold for these values of parameters, indeed

$\mathbf{H 1} a_{2} e-d_{2}=3>0$,

$\mathbf{H} 2 a_{1} b_{2} d_{2}-k=1>0$,

H3 $a_{1}^{2} b_{2}^{2} d_{2}^{2}-4 b_{1}\left(d_{2}-a_{2} e\right)^{2} \rho^{2}=1 \geq 0$,

H4 $a_{1} b_{2} c d_{2}-2 b_{2} d d_{2}-c k=8 \rho\left(-1+\frac{32}{32+243 \rho^{3} \omega^{2}}\right)>0$,

H5 $-a_{2}^{2} b_{2}^{2} e^{2} k B_{2}^{2}\left(a_{1} a_{2} b_{2} d_{2} e-B_{1} k\right)+4 d_{2} B_{1}^{6} \rho^{2} \omega^{2}=-704+2916 \rho^{2} \omega^{2}>0$.

And the first Lyapunov coefficient $\ell\left(p_{1}\right)$ is

$$
\ell\left(p_{1}\right)=\frac{l_{a}}{l_{b}}
$$

Where

$$
\begin{aligned}
& l_{a}=-\left(8192(-1356+22295 \rho)+512 \rho^{3}(4076159+4054698 \rho) \omega^{2}+51018336 \rho^{6}(285+101 \rho) \omega^{4}+3486784401 \rho^{9} \omega^{6}\right) . \\
& I_{b}=648 \omega\left(4096+\rho^{2}\left(16384+243 \rho \omega^{2}\left(256\left(1+4 \rho\left(3+\rho+3 \rho^{2}\right)\right)+972 \rho^{3}(1+4 \rho(12+37 \rho)) \omega^{2}+177147 \rho^{7} \omega^{4}\right)\right)\right) .
\end{aligned}
$$

We have that $I_{b}>0$ for any $\rho$ and $\omega$. Note that $I_{a}<0$ if

$$
\rho>\frac{1356}{22295} .
$$




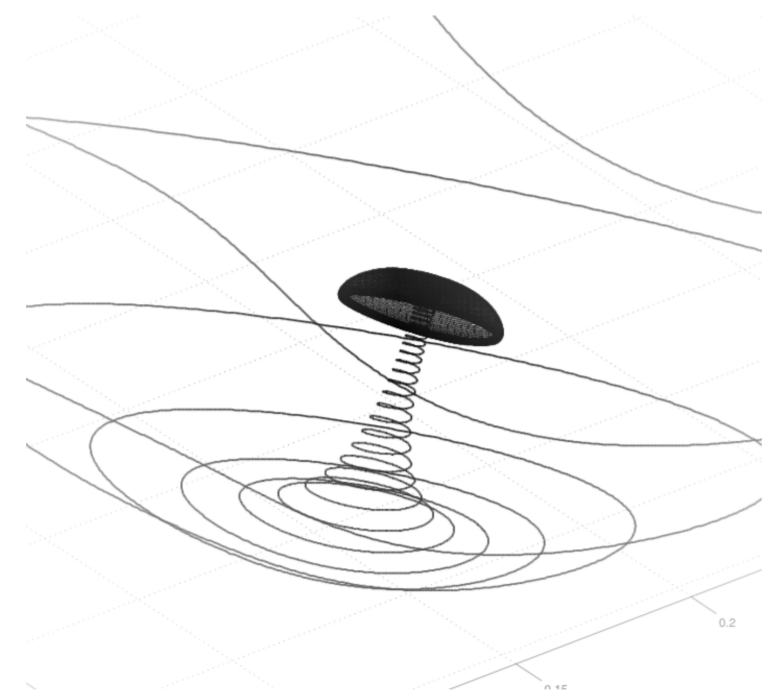

Figure 1. We show an orbit tending to the local attractor definided by a stable Hopf periodic orbit.

Then condition (11) holds if $\omega>\frac{22295 \sqrt{11}}{9153}$, and then we obtain that the first Lyapunov coefficient $\ell\left(p_{1}\right)$ is negative for all the previous values of $\rho$ and $\omega$. In the particular case that $\rho=\omega=1$ the value of $\ell\left(p_{1}\right)$ is

$$
\ell\left(p_{1}\right)=-\frac{27514393169}{59228395560}
$$

Recall that when $\ell\left(p_{1}\right)$ is negative we have a subcritical Hopf bifurcation and then the periodic orbit obtained of the bifurcation is stable in the planar central surface, and since the real eigenvalue $\alpha$ is negative, then the limit cycle living in the central surface is a local attractor.

In Figure 1 we exhibit the local attractor defined by the stable limit cycle of differential systems (2) with parameters values $a_{1}=1, a_{2}=2, b_{2}=2, d_{2}=1, e=2, k=1, \rho=1, \omega=1$ and $\epsilon=0.1$. The singular points are $p_{1}=(1 / 6,2 / 3,648 / 275)$ and $p_{2}=(1 / 2,2 / 3,22707 / 1100)$. The eigenvalues of linear approximation of system at $p_{2}$ are $\{1.8292+3.82836 i, 1.8292-$ $3.82836 i, 0.226503\}$ then it is a repellor.

\section{Appendix}

The bilinear function $B$ at vector $(x, y, z)$ is given by

$$
B((x, y, z),(x, y, z))=\left(B_{11}, B_{12}, B_{13}\right),
$$

where

$$
\begin{aligned}
& B_{11}=-\frac{a_{1} B_{2}^{2} y^{2}+2 B_{2}\left(2 a_{1} a_{2} b_{2} e+k\right) x y \rho-2 a_{2} b_{2} e\left(a_{1} b_{2} d_{2}-3 k\right) x^{2} \rho^{2}}{2 B_{1}^{2} \rho^{2}} ; \\
& B_{12}=-\frac{s_{1}}{s_{2}} ; \\
& B_{13}=-\frac{B_{2} y\left(a_{1}^{3} a_{2}^{2} b_{2}^{4} d_{2}^{4} e^{2} k B_{2}^{2} z-4 B_{1}^{5} \rho^{3}\left(a_{1}^{2} b_{2} d_{2}^{3} z+2 a_{2} e^{2} k x \rho^{2}\right) \omega^{2}\right)}{a_{1} d_{2}^{2} B_{1} \rho^{2}\left(a_{1} a_{2}^{2} b_{2}^{3} d_{2} e^{2} k B_{2}^{2}-4 B_{1}^{5} \rho^{3} \omega^{2}\right)} ;
\end{aligned}
$$




$$
\begin{aligned}
s_{1}= & a_{1}^{9} a_{2}^{5} b_{2}^{12} d_{2}^{9} e^{4} k y z-6 a_{1}^{8} a_{2}^{5} b_{2}^{11} d_{2}^{8} e^{4} k^{2} y z+15 a_{1}^{7} a_{2}^{5} b_{2}^{10} d_{2}^{7} e^{4} k^{3} y z \\
& -20 a_{1}^{6} a_{2}^{5} b_{2}^{9} d_{2}^{6} e^{4} k^{4} y z+15 a_{1}^{5} a_{2}^{5} b_{2}^{8} d_{2}^{5} e^{4} k^{5} y z-6 a_{1}^{4} a_{2}^{5} b_{2}^{7} d_{2}^{4} e^{4} k^{6} y z \\
& +a_{1}^{3} a_{2}^{5} b_{2}^{6} d_{2}^{3} e^{4} k^{7} y z+2 a_{1}^{8} a_{2}^{5} b_{2}^{12} d_{2}^{9} e^{4} k x z \rho-12 a_{1}^{7} a_{2}^{5} b_{2}^{11} d_{2}^{8} e^{4} k^{2} x z \rho \\
& +30 a_{1}^{6} a_{2}^{5} b_{2}^{10} d_{2}^{7} e^{4} k^{3} x z \rho-40 a_{1}^{5} a_{2}^{5} b_{2}^{9} d_{2}^{6} e^{4} k^{4} x z \rho+30 a_{1}^{4} a_{2}^{5} b_{2}^{8} d_{2}^{5} e^{4} k^{5} x z \rho \\
& -12 a_{1}^{3} a_{2}^{5} b_{2}^{7} d_{2}^{4} e^{4} k^{6} x z \rho+2 a_{1}^{2} a_{2}^{5} b_{2}^{6} d_{2}^{3} e^{4} k^{7} x z \rho-4 a_{2}^{2} b_{2}^{2} e^{2} B_{1}^{5} B_{2}^{2} \rho^{3} s_{3} \omega^{2} \\
& +32 B_{1}^{11} x \rho^{7}\left(\left(d_{2}+a_{2} e\right) B_{2} y+a_{2} b_{2} d_{2} e x \rho\right) \omega^{4} ; \\
s_{2}= & a_{1} a_{2}^{2} b_{2}^{2} d_{2} e^{2} B_{1}^{2} B_{2}^{3} \rho^{2}\left(a_{1} a_{2}^{2} b_{2}^{3} d_{2} e^{2} k B_{2}^{2}-4 B_{1}^{5} \rho^{3} \omega^{2}\right) ; \\
s_{3}= & a_{1}^{2} a_{2} b_{2} d_{2}^{2} B_{2}^{2} y z+a_{1} a_{2} B_{2}^{2}\left(-e k y^{2}+2 b_{2} d_{2}^{2} x z\right) \rho-2 d_{2} B_{2} k D_{1} x y \rho^{2} \\
& -2 a_{2} b_{2} d_{2} e k^{2} x^{2} \rho^{3} .
\end{aligned}
$$

The trilinear function $C$ at vector $(x, y, z)$ is given by

$$
C((x, y, z),(x, y, z),(x, y, z))=\left(C_{11}, C_{12}, C_{13}\right),
$$

where

$$
\begin{aligned}
& C_{11}=-\frac{3 x\left(2 a_{1}\left(-B_{2}\right) y^{2}+\left(a_{1} b_{2}\left(d_{2}-2 a_{2} e\right)-3 k\right) x y \rho+2 a_{2} b_{2} e x^{2} \rho^{2}\right)}{B_{1} \rho} ; \\
& C_{12}=-\frac{s_{4}}{s_{5}} ; \\
& C_{13}=\frac{6 x y\left(a_{1}^{2} a_{2}^{2} b_{2}^{3} d_{2}^{3} e^{2} B_{2}^{3} k z-4 B_{1}^{5} \rho^{3}\left(a_{1} d_{2}^{2} B_{2} z+a_{2} e^{2} k x \rho^{2}\right) \omega^{2}\right)}{a_{1} d_{2}^{2} \rho\left(a_{1} a_{2}^{2} b_{2}^{3} d_{2} e^{2} k B_{2}^{2}-4 B_{1}^{5} \rho^{3} \omega^{2}\right)} ;
\end{aligned}
$$

$$
\begin{aligned}
s_{4}= & 6 x s_{7} ; \\
s_{5}= & \left(a_{1} a_{2}^{2} b_{2}^{3} d_{2}^{2} e^{2} B_{1} B_{2}^{3} \rho\left(-a_{1} a_{2}^{2} b_{2}^{3} d_{2} e^{2} k B_{2}^{2}+4 B_{1}^{5} \rho^{3} \omega^{2}\right)\right) ; \\
s_{6}= & a_{1} a_{2} b_{2} d_{2}^{2} B_{2}^{2} y z+B_{2}\left(k\left(-a_{1} a_{2} b_{2} d_{2} e-d_{2} k+a_{2} e k\right) y^{2}+a_{1} a_{2} b_{2}^{2} d_{2}^{3} x z\right) \rho \\
& -b_{2} d_{2}^{2} k\left(a_{1} a_{2} b_{2} e+k\right) x y \rho^{2} ; \\
s_{7}= & a_{1}^{8} a_{2}^{5} b_{2}^{12} d_{2}^{9} e^{4} k y z-6 a_{1}^{7} a_{2}^{5} b_{2}^{11} d_{2}^{8} e^{4} k^{2} y z+15 a_{1}^{6} a_{2}^{5} b_{2}^{10} d_{2}^{7} e^{4} k^{3} y z \\
& -20 a_{1}^{5} a_{2}^{5} b_{2}^{9} d_{2}^{6} e^{4} k^{4} y z+15 a_{1}^{4} a_{2}^{5} b_{2}^{8} d_{2}^{5} e^{4} k^{5} y z-6 a_{1}^{3} a_{2}^{5} b_{2}^{7} d_{2}^{4} e^{4} k^{6} y z \\
& +a_{1}^{2} a_{2}^{5} b_{2}^{6} d_{2}^{3} e^{4} k^{7} y z+a_{1}^{7} a_{2}^{5} b_{2}^{12} d_{2}^{9} e^{4} k x z \rho-5 a_{1}^{6} a_{2}^{5} b_{2}^{11} d_{2}^{8} e^{4} k^{2} x z \rho \\
& +10 a_{1}^{5} a_{2}^{5} b_{2}^{10} d_{2}^{7} e^{4} k^{3} x z \rho-10 a_{1}^{4} a_{2}^{5} b_{2}^{9} d_{2}^{6} e^{4} k^{4} x z \rho+5 a_{1}^{3} a_{2}^{5} b_{2}^{8} d_{2}^{5} e^{4} k^{5} x z \rho \\
& -a_{1}^{2} a_{2}^{5} b_{2}^{7} d_{2}^{4} e^{4} k^{6} x z \rho+4 a_{2}^{2} b_{2}^{2} e^{2}\left(-B_{1}\right)^{5} B_{2}^{2} \rho^{3} s_{6} \omega^{2} \\
& +16 d_{2} B_{1}^{11} y \rho^{6}\left(a_{1} b_{2} d_{2} y-k y+b_{2}\left(d_{2}+a_{2} e\right) \times \rho\right) \omega^{4} .
\end{aligned}
$$

The eigenvector $q=\left(q_{11}, q_{12}, q_{13}\right)$ of $A$ corresponding to eigenvalue $i \omega$ have the coordinates

$$
\begin{aligned}
& q_{11}=\frac{a_{1} d_{2} B_{2} r_{3}}{e B_{1}^{3} k \rho^{3} \omega r_{0}} \sqrt{\frac{r_{2}}{r_{1}}} ; \\
& q_{12}=-\frac{2 i d_{2} r_{3}}{a_{2} b_{2} e^{2} B_{1}^{3} B_{2} k \rho^{2} \omega} \sqrt{\frac{r_{2}}{r_{1}}} ; \\
& q_{13}=4 \sqrt{\frac{r_{2}}{r_{1}}} ;
\end{aligned}
$$




$$
\begin{aligned}
r_{1}= & a_{1}^{12} a_{2}^{6} b_{2}^{16} d_{2}^{12} e^{6} k^{2}-8 a_{1}^{11} a_{2}^{6} b_{2}^{15} d_{2}^{11} e^{6} k^{3}+28 a_{1}^{10} a_{2}^{6} b_{2}^{14} d_{2}^{10} e^{6} k^{4}-56 a_{1}^{9} a_{2}^{6} b_{2}^{13} d_{2}^{9} e^{6} k^{5} \\
& +70 a_{1}^{8} a_{2}^{6} b_{2}^{12} d_{2}^{8} e^{6} k^{6}-56 a_{1}^{7} a_{2}^{6} b_{2}^{11} d_{2}^{7} e^{6} k^{7}+28 a_{1}^{6} a_{2}^{6} b_{2}^{10} d_{2}^{6} e^{6} k^{8}-8 a_{1}^{5} a_{2}^{6} b_{2}^{9} d_{2}^{5} e^{6} k^{9} \\
& +a_{1}^{4} a_{2}^{6} b_{2}^{8} d_{2}^{4} e^{6} k^{10}+4 a_{1}^{8} a_{2}^{6} b_{2}^{14} d_{2}^{10} e^{6} k^{4} \rho^{2}-24 a_{1}^{7} a_{2}^{6} b_{2}^{13} d_{2}^{9} e^{6} k^{5} \rho^{2}+60 a_{1}^{6} a_{2}^{6} b_{2}^{12} d_{2}^{8} e^{6} k^{6} \rho^{2} \\
& -80 a_{1}^{5} a_{2}^{6} b_{2}^{11} d_{2}^{7} e^{6} k^{7} \rho^{2}+60 a_{1}^{4} a_{2}^{6} b_{2}^{10} d_{2}^{6} e^{6} k^{8} \rho^{2}-24 a_{1}^{3} a_{2}^{6} b_{2}^{9} d_{2}^{5} e^{6} k^{9} \rho^{2} \\
+ & 4 a_{1}^{2} a_{2}^{6} b_{2}^{8} d_{2}^{4} e^{6} k^{10} \rho^{2}+8 a_{2}^{4} b_{2}^{4} e^{4}\left(-B_{1}\right)^{5} k B_{2}^{4} \rho^{3} r_{11} ; \\
r_{11}= & \left(a_{1}^{3} b_{2} d_{2}^{3} B_{2}^{2}-2 a_{1}^{2} b_{2}^{2} d_{2}^{4} B_{1} k \rho+4 a_{1} b_{2} d_{2}^{3} k^{2} \rho^{2}+2 e^{2}\left(-B_{1}\right) k^{3} \rho^{3}\right) \omega^{2} \\
& +16 a_{2}^{2} b_{2}^{2} e^{2} B_{1}^{10} B_{2}^{2} \rho^{6}\left(a_{1}^{2} d_{2}^{2} B_{2}^{2}-8 a_{1} b_{2} d_{2}^{3} B_{1} k \rho+4\left(-2 a_{2} d_{2} e^{3}+a_{2}^{2} e^{4}\right.\right. \\
& \left.\left.+d_{2}^{2}\left(1+e^{2}\right)\right) k^{2} \rho^{2}\right) \omega^{4}+256 d_{2}^{2} B_{1}^{16} \rho^{10} \omega^{6} \\
r_{0}= & -i a_{1} a_{2} b_{2}^{2} d_{2} e k-2 d_{2}^{3} \rho \omega-6 a_{2}^{2} d_{2} e^{2} \rho \omega+2 a_{2}^{3} e^{3} \rho \omega+a_{2} e\left(i b_{2} k^{2}+6 d_{2}^{2} \rho \omega\right) ; \\
r_{2}= & a_{2}^{2} b_{2}^{2} e^{4} B_{1}^{6} k^{2} B_{2}^{2} \rho^{6} \omega^{2}\left(a_{2}^{2} b_{2}^{2} e^{2} k^{2} B_{2}^{2}+4 B_{1}^{6} \rho^{2} \omega^{2}\right) ; \\
r_{3}= & a_{1}^{3} a_{2}^{2} b_{2}^{5} d_{2}^{3} e^{2} k-2 a_{1}^{2} a_{2}^{2} b_{2}^{4} d_{2}^{2} e^{2} k^{2}+a_{1} a_{2}^{2} b_{2}^{3} d_{2} e^{2} k^{3}-4 B_{1}^{5} \rho^{3} \omega^{2} ;
\end{aligned}
$$

The adjoint eigenvector $p=\left(p_{11}, p_{12}, p_{13}\right)$ of transpose matrix $A$ corresponding to the eigenvalue $-i \omega$ have the coordinates

$$
\begin{aligned}
& p_{11}=\frac{16 e B_{1}^{8} k B_{2}^{2} \rho^{6} \omega^{3} g_{0}\left(i a_{2} b_{2} e B_{2} k+2 B_{1}^{3} \rho \omega\right)}{a_{1} d_{2} B_{2}^{3}\left(a_{1} a_{2}^{2} b_{2}^{3} d_{2} e^{2} k B_{2}^{2}-4 B_{1}^{5} \rho^{3} \omega^{2}\right) g_{3}} \sqrt{\frac{g_{1}}{g_{2}},} \\
& p_{12}=-\frac{2 i a_{2} b_{2} e^{2} B_{1}^{3} k B_{2}^{2} \rho^{2} \omega g_{0}}{d_{2} B_{2} g_{3}} \sqrt{\frac{g_{1}}{g_{2}}}, \\
& p_{1} 3=\frac{a_{1} a_{2}^{2} b_{2}^{3} d_{2} e^{2} k B_{2}^{2} g_{0}}{g_{3}} \sqrt{\frac{g_{1}}{g_{2}}}
\end{aligned}
$$

$$
\begin{aligned}
g_{0}= & i_{2} b_{2} e B_{2} k-2\left(B_{1}\right)^{3} \rho \omega ; \\
g_{1}= & a_{1}^{12} a_{2}^{6} b_{2}^{16} d_{2}^{12} e^{6} k^{2}-8 a_{1}^{11} a_{2}^{6} b_{2}^{15} d_{2}^{11} e^{6} k^{3}+28 a_{1}^{10} a_{2}^{6} b_{2}^{14} d_{2}^{10} e^{6} k^{4} \\
& -56 a_{1}^{9} a_{2}^{6} b_{2}^{13} d_{2}^{9} e^{6} k^{5}+70 a_{1}^{8} a_{2}^{6} b_{2}^{12} d_{2}^{8} e^{6} k^{6}-56 a_{1}^{7} a_{2}^{6} b_{2}^{11} d_{2}^{7} e^{6} k^{7} \\
& +28 a_{1}^{6} a_{2}^{6} b_{2}^{10} d_{2}^{6} e^{6} k^{8}-8 a_{1}^{5} a_{2}^{6} b_{2}^{9} d_{2}^{5} e^{6} k^{9}+a_{1}^{4} a_{2}^{6} b_{2}^{8} d_{2}^{4} e^{6} k^{10} \\
& +4 a_{1}^{8} a_{2}^{6} b_{2}^{14} d_{2}^{10} e^{6} k^{4} \rho^{2}-24 a_{1}^{7} a_{2}^{6} b_{2}^{13} d_{2}^{9} e^{6} k^{5} \rho^{2}+60 a_{1}^{6} a_{2}^{6} b_{2}^{12} d_{2}^{8} e^{6} k^{6} \rho^{2} \\
& -80 a_{1}^{5} a_{2}^{6} b_{2}^{11} d_{2}^{7} e^{6} k^{7} \rho^{2}+60 a_{1}^{4} a_{2}^{6} b_{2}^{10} d_{2}^{6} e^{6} k^{8} \rho^{2}-24 a_{1}^{3} a_{2}^{6} b_{2}^{9} d_{2}^{5} e^{6} k^{9} \rho^{2} \\
& +4 a_{1}^{2} a_{2}^{6} b_{2}^{8} d_{2}^{4} e^{6} k^{10} \rho^{2}+8 a_{2}^{4} b_{2}^{4} e^{4}\left(-B_{1}\right)^{5} k B_{2}^{4} \rho^{3}\left(a_{1}^{3} b_{2} d_{2}^{3} B_{2}^{2}\right. \\
& \left.-2 a_{1}^{2} b_{2}^{2} d_{2}^{4}\left(B_{1}\right) k \rho+4 a_{1} b_{2} d_{2}^{3} k^{2} \rho^{2}+2 e^{2}\left(-B_{1}\right) k^{3} \rho^{3}\right) \omega^{2} \\
& +16 a_{2}^{2} b_{2}^{2} e^{2}\left(B_{1}\right)^{10}\left(-B_{2}\right)^{2} \rho^{6}\left(a_{1}^{2} d_{2}^{2}\left(-B_{2}\right)^{2}-8 a_{1} b_{2} d_{2}^{3}\left(B_{1}\right) k \rho\right. \\
& \left.+4\left(-2 a_{2} d_{2} e^{3}+a_{2}^{2} e^{4}+d_{2}^{2}\left(1+e^{2}\right)\right) k^{2} \rho^{2}\right) \omega^{4} \\
& +256 d_{2}^{2}\left(B_{1}\right)^{16} \rho^{10} \omega^{6} ; \\
g_{2}= & a_{2}^{2} b_{2}^{2} e^{4}\left(B_{1}\right)^{6} k^{2}\left(-B_{2}\right)^{2} \rho^{6} \omega^{2}\left(a_{2}^{2} b_{2}^{2} e^{2} k^{2}\left(-B_{2}\right)^{2}+4\left(B_{1}\right)^{6} \rho^{2} \omega^{2}\right) ; \\
g_{3}= & 8 i a_{1} a_{2}^{3} b_{2}^{4} d_{2} e^{3} B_{2}^{3} k^{2}+16 a_{1} a_{2}^{2} b_{2}^{3} d_{2} e^{2}\left(-B_{1}\right)^{3} k\left(-B_{2}\right)^{2} \rho \omega \\
& +64\left(B_{1}\right)^{8} \rho^{4} \omega^{3} .
\end{aligned}
$$

The expression of the first Lyapunov coefficient $\ell\left(p_{1}\right)$, at the equillibrium point $p_{1}$.

$$
\ell\left(p_{1}\right)=\frac{l_{a}}{l_{b}} .
$$


The expression of numerator of $\ell\left(p_{1}\right)$, is $l_{a}$

$$
\begin{aligned}
l_{a}= & a_{1} d_{2}\left(l_{a 1}+a_{1}^{8} a_{2}^{5} b_{2}^{12} d_{2}^{8} e^{5} k l_{a 2}+a_{1}^{6} a_{2}^{3} b_{2}^{10} d_{2}^{6} e^{3} k^{3} l_{a 3}+a_{1}^{7} a_{2}^{4} b_{2}^{11} d_{2}^{7} e^{4} k^{2} l_{a 4}\right. \\
& +24\left(d_{2}-a_{2} e\right)^{7} k \rho^{4} \omega^{2} l_{a 5}+a_{1}^{2} a_{2}^{2} b_{2}^{4} d_{2}^{2} e^{2} k^{3} l_{a 6}+a_{1}^{3} a_{2}^{2} b_{2}^{5} d_{2}^{3} e^{2} k^{2} l_{a} \\
& \left.-a_{1}^{5} a_{2}^{3} b_{2}^{7} d_{2}^{5} e^{3} l_{a 8}+a_{1}^{4} a_{2}^{2} b_{2}^{6} d_{2}^{4} e^{2} k l_{a 9}+l_{a 10}\right) .
\end{aligned}
$$

The expression of denominator of $\ell\left(p_{1}\right)$, is $I_{b}$

$$
\begin{aligned}
I_{b}= & 6\left(d_{2}-a_{2} e\right)^{3} \omega\left(I_{b 1}+8 a_{2}^{4} b_{2}^{4} e^{4}\left(-d_{2}+a_{2} e\right)^{5} k\left(-a_{1} b_{2} d_{2}+k\right)^{4} \rho^{3} I_{b 2} \omega^{2}\right. \\
& +16 a_{2}^{2} b_{2}^{2} e^{2}\left(d_{2}-a_{2} e\right)^{10}\left(-a_{1} b_{2} d_{2}+k\right)^{2} \rho^{6} I_{b 3} \omega^{4} \\
& \left.+256 d_{2}^{2}\left(d_{2}-a_{2} e\right)^{16} \rho^{10} \omega^{6}\right) .
\end{aligned}
$$

Where,

$$
\begin{aligned}
& l_{a 1}=a_{1}^{10} a_{2}^{6} b_{2}^{16} d_{2}^{10} e^{6}\left(d_{2}-a_{2} e\right)^{2} k^{3}\left(10 d_{2}\left(d_{2}-a_{2} e\right)-3 a_{2} e \rho\right) \\
& +a_{1}^{9} a_{2}^{6} b_{2}^{15} d_{2}^{9} e^{6}\left(d_{2}-a_{2} e\right)^{2} k^{4}\left(-80 d_{2}\left(d_{2}-a_{2} e\right)+\left(d_{2}+6 a_{2} e\right) \rho\right), \\
& l_{a 2}=b_{2}^{2} k^{4}\left(a_{2} d_{2}^{3} e\left(280 d_{2}-13 \rho\right)+3 a_{2}^{3} d_{2} e^{3}\left(280 d_{2}-\rho\right)+2 d_{2}^{4} \rho\right. \\
& \left.-a_{2}^{4} e^{4}\left(280 d_{2}+3 \rho\right)+a_{2}^{2} d_{2}^{2} e^{2}\left(-876 d_{2}+5 \rho\right)\right)+36\left(d_{2}-a_{2} e\right)^{7} \rho^{4} \omega^{2} \text {, } \\
& l_{a 3}=a_{2}^{2} b_{2}^{2} e^{2} k^{4}\left(7 a_{2} d_{2}^{3} e\left(100 d_{2}-23 \rho\right)+42 d_{2}^{4} \rho-5 a_{2}^{4} e^{4}\left(140 d_{2}+93 \rho\right)\right. \\
& \left.-a_{2}^{2} d_{2}^{2} e^{2}\left(2418 d_{2}+457 \rho\right)+a_{2}^{3} d_{2} e^{3}\left(2100 d_{2}+853 \rho\right)\right)+4 \rho^{3}\left(2 d_{2}^{10}\right. \\
& +51 a_{2} d_{2}^{9} e-444 a_{2}^{2} d_{2}^{8} e^{2}+1476 a_{2}^{3} d_{2}^{7} e^{3}-3024 a_{2}^{4} d_{2}^{6} e^{4} \\
& +2562 a_{2}^{5} d_{2}^{5} e^{5}-1176 a_{2}^{6} d_{2}^{4} e^{6}-156 a_{2}^{7} d_{2}^{3} e^{7}+442 a_{2}^{8} d_{2}^{2} e^{8} \\
& \left.-221 a_{2}^{9} d_{2} e^{9}+36 a_{2}^{10} e^{10}+3 a_{2} e\left(d_{2}-a_{2} e\right)^{7}\left(10 d_{2}+9 a_{2} e\right) \rho\right) \omega^{2} \\
& l_{a 4}=a_{2} b_{2}^{2} e k^{4}\left(-14 d_{2}^{4} \rho+7 a_{2} d_{2}^{3} e\left(-80 d_{2}+9 \rho\right)+10 a_{2}^{4} e^{4}\left(56 d_{2}+15 \rho\right)\right. \\
& \left.+6 a_{2}^{2} d_{2}^{2} e^{2}\left(308 d_{2}+23 \rho\right)-5 a_{2}^{3} d_{2} e^{3}\left(336 d_{2}+53 \rho\right)\right) \\
& -4 \rho^{3}\left(11 d_{2}^{9}-82 a_{2} d_{2}^{8} e+260 a_{2}^{2} d_{2}^{7} e^{2}-504 a_{2}^{3} d_{2}^{6} e^{3}+434 a_{2}^{4} d_{2}^{5} e^{4}\right. \\
& -196 a_{2}^{5} d_{2}^{4} e^{5}-28 a_{2}^{6} d_{2}^{3} e^{6}+72 a_{2}^{7} d_{2}^{2} e^{7}-37 a_{2}^{8} d_{2} e^{8} \\
& \left.+6 a_{2}^{9} e^{9}+3\left(d_{2}-a_{2} e\right)^{7}\left(3 d_{2}+10 a_{2} e\right) \rho\right) \omega^{2} \\
& l_{a 5}=-a_{2}^{5} b_{2}^{4} e^{5} k^{8}+4 a_{2}^{2} b_{2}^{2} e^{2}\left(d_{2}-a_{2} e\right)^{5} k^{4} \rho^{2}\left(-a_{2}^{2} e^{2}+a_{2} e\left(d_{2}-8 \rho\right)\right. \\
& \left.+5 d_{2} \rho\right) \omega^{2}+8\left(d_{2}-a_{2} e\right)^{11}\left(d_{2}+a_{2} e\right) \rho^{5} \omega^{4} \text {, } \\
& l_{a 6}=a_{2}^{3} b_{2}^{4} e^{3} k^{8}\left(a_{2} d_{2}^{3} e\left(10 d_{2}-19 \rho\right)+6 d_{2}^{4} \rho-3 a_{2}^{2} d_{2}^{2} e^{2}\left(12 d_{2}+17 \rho\right)\right. \\
& \left.+5 a_{2}^{3} d_{2} e^{3}\left(6 d_{2}+19 \rho\right)-a_{2}^{4} e^{4}\left(10 d_{2}+51 \rho\right)\right)+4 a_{2} b_{2}^{2} e k^{4} \rho^{3}\left(2 d_{2}^{10}\right. \\
& +55 a_{2} d_{2}^{9} e-476 a_{2}^{2} d_{2}^{8} e^{2}+1588 a_{2}^{3} d_{2}^{7} e^{3}-3024 a_{2}^{4} d_{2}^{6} e^{4} \\
& +2842 a_{2}^{5} d_{2}^{5} e^{5}-1400 a_{2}^{6} d_{2}^{4} e^{6}-44 a_{2}^{7} d_{2}^{3} e^{7}+442 a_{2}^{8} d_{2}^{2} e^{8} \\
& \left.-217 a_{2}^{9} d_{2} e^{9}+36 a_{2}^{10} e^{10}+3 a_{2} e\left(32 d_{2}-57 a_{2} e\right)\left(d_{2}-a_{2} e\right)^{7} \rho\right) \omega^{2} \\
& -48\left(d_{2}-3 a_{2} e\right)\left(d_{2}-a_{2} e\right)^{13} \rho^{6} \omega^{4} \text {, }
\end{aligned}
$$




$$
\begin{aligned}
& l_{a 7}=a_{2}^{3} b_{2}^{4} e^{3} k^{8}\left(-30 d_{2}^{4} \rho+12 a_{2}^{2} d_{2}^{2} e^{2}\left(24 d_{2}+7 \rho\right)+4 a_{2}^{4} e^{4}\left(20 d_{2}+21 \rho\right)\right. \\
& \left.+a_{2} d_{2}^{3} e\left(-80 d_{2}+97 \rho\right)-a_{2}^{3} d_{2} e^{3}\left(240 d_{2}+131 \rho\right)\right) \\
& -4 a_{2} b_{2}^{2} e k^{4} \rho^{3}\left(8 d_{2}^{10}+111 a_{2} d_{2}^{9} e-1122 a_{2}^{2} d_{2}^{8} e^{2}\right. \\
& +4020 a_{2}^{3} d_{2}^{7} e^{3}-8568 a_{2}^{4} d_{2}^{6} e^{4}+8778 a_{2}^{5} d_{2}^{5} e^{5}-5796 a_{2}^{6} d_{2}^{4} e^{6} \\
& +1812 a_{2}^{7} d_{2}^{3} e^{7}+112 a_{2}^{8} d_{2}^{2} e^{8}-257 a_{2}^{9} d_{2} e^{9}+54 a_{2}^{10} e^{10} \\
& \left.+3 a_{2} e\left(34 d_{2}-121 a_{2} e\right)\left(d_{2}-a_{2} e\right)^{7} \rho\right) \omega^{2} \\
& +48\left(d_{2}-a_{2} e\right)^{13}\left(5 d_{2}-11 a_{2} e-4 \rho\right) \rho^{6} w^{4}, \\
& l_{a 8}=a_{2}^{2} b_{2}^{4} e^{2} k^{8}\left(a_{2} d_{2}^{3} e\left(560 d_{2}-239 \rho\right)+68 d_{2}^{4} \rho-80 a_{2}^{4} e^{4}\left(7 d_{2}+6 \rho\right)\right. \\
& \left.-2 a_{2}^{2} d_{2}^{2} e^{2}\left(996 d_{2}+239 \rho\right)+a_{2}^{3} d_{2} e^{3}\left(1680 d_{2}+857 \rho\right)\right) \\
& +4 b_{2}^{2} k^{4} \rho^{3}\left(2 \left(4 d_{2}^{10}+53 a_{2} d_{2}^{9} e-541 a_{2}^{2} d_{2}^{8} e^{2}+1940 a_{2}^{3} d_{2}^{7} e^{3}\right.\right. \\
& -4284 a_{2}^{4} d_{2}^{6} e^{4}+4214 a_{2}^{5} d_{2}^{5} e^{5}-2758 a_{2}^{6} d_{2}^{4} e^{6}+836 a_{2}^{7} d_{2}^{3} e^{7} \\
& \left.+56 a_{2}^{8} d_{2}^{2} e^{8}-131 a_{2}^{9} d_{2} e^{9}+27 a_{2}^{10} e^{10}\right) \\
& \left.+3 a_{2} e\left(11 d_{2}-3 a_{2} e\right)\left(d_{2}-a_{2} e\right)^{7} \rho\right) \omega^{2} \\
& +144\left(d_{2}-a_{2} e\right)^{12} \rho^{7} \omega^{4} \text {, } \\
& l_{a 9}=a_{2}^{3} b_{2}^{4} e^{3} k^{8}\left(a_{2} d_{2}^{3} e\left(280 d_{2}-207 \rho\right)+62 d_{2}^{4} \rho-12 a_{2}^{2} d_{2}^{2} e^{2}\left(84 d_{2}+17 \rho\right)\right. \\
& \left.-4 a_{2}^{4} e^{4}\left(70 d_{2}+51 \rho\right)+5 a_{2}^{3} d_{2} e^{3}\left(168 d_{2}+65 \rho\right)\right) \\
& +4 a_{2} b_{2}^{2} e k^{4} \rho^{3}\left(2 \left(6 d_{2}^{10}+67 a_{2} d_{2}^{9} e-728 a_{2}^{2} d_{2}^{8} e^{2}+2692 a_{2}^{3} d_{2}^{7} e^{3}\right.\right. \\
& -6048 a_{2}^{4} d_{2}^{6} e^{4}+6370 a_{2}^{5} d_{2}^{5} e^{5}-4592 a_{2}^{6} d_{2}^{4} e^{6}+1828 a_{2}^{7} d_{2}^{3} e^{7} \\
& \left.-258 a_{2}^{8} d_{2}^{2} e^{8}-77 a_{2}^{9} d_{2} e^{9}+24 a_{2}^{10} e^{10}\right) \\
& \left.+3 a_{2} e\left(16 d_{2}-79 a_{2} e\right)\left(d_{2}-a_{2} e\right)^{7} \rho\right) \omega^{2} \\
& -48\left(d_{2}-a_{2} e\right)^{12} \rho^{6}\left(3\left(d_{2}-a_{2} e\right)^{2}-\left(3 d_{2}+5 a_{2} e\right) \rho\right) \omega^{4}, \\
& l_{a 10}=6 a_{1} b_{2} d_{2}\left(d_{2}-a_{2} e\right)^{2} \rho\left(a_{2}^{7} b_{2}^{6} e^{7} k^{12}-2 a_{2}^{4} b_{2}^{4} e^{4}\left(-d_{2}+a_{2} e\right)^{5} k^{8} \rho^{2}\left(-4 d_{2}^{2}\right.\right. \\
& \left.+2 d_{2}\left(a_{2} e-5 \rho\right)+a_{2} e\left(2 a_{2} e+29 \rho\right)\right) \omega^{2} \\
& -8 a_{2}^{2} b_{2}^{2} e^{2}\left(d_{2}-a_{2} e\right)^{10} k^{4} \rho^{5}\left(d_{2}^{2}+4 d_{2}\left(2 a_{2} e+3 \rho\right)\right. \\
& \left.\left.-a_{2} e\left(9 a_{2} e+25 \rho\right)\right) \omega^{4}+32\left(d_{2}-a_{2} e\right)^{16}\left(3 d_{2}-a_{2} e\right) \rho^{8} \omega^{6}\right) \text {. } \\
& I_{b 1}=a_{1}^{12} a_{2}^{6} b_{2}^{16} d_{2}^{12} e^{6} k^{2}-8 a_{1}^{11} a_{2}^{6} b_{2}^{15} d_{2}^{11} e^{6} k^{3}+28 a_{1}^{10} a_{2}^{6} b_{2}^{14} d_{2}^{10} e^{6} k^{4} \\
& -56 a_{1}^{9} a_{2}^{6} b_{2}^{13} d_{2}^{9} e^{6} k^{5}+70 a_{1}^{8} a_{2}^{6} b_{2}^{12} d_{2}^{8} e^{6} k^{6}-56 a_{1}^{7} a_{2}^{6} b_{2}^{11} d_{2}^{7} e^{6} k^{7} \\
& +28 a_{1}^{6} a_{2}^{6} b_{2}^{10} d_{2}^{6} e^{6} k^{8}-8 a_{1}^{5} a_{2}^{6} b_{2}^{9} d_{2}^{5} e^{6} k^{9}+a_{1}^{4} a_{2}^{6} b_{2}^{8} d_{2}^{4} e^{6} k^{10} \\
& +4 a_{1}^{8} a_{2}^{6} b_{2}^{14} d_{2}^{10} e^{6} k^{4} \rho^{2}-24 a_{1}^{7} a_{2}^{6} b_{2}^{13} d_{2}^{9} e^{6} k^{5} \rho^{2}+60 a_{1}^{6} a_{2}^{6} b_{2}^{12} d_{2}^{8} e^{6} k^{6} \rho^{2} \\
& -80 a_{1}^{5} a_{2}^{6} b_{2}^{11} d_{2}^{7} e^{6} k^{7} \rho^{2}+60 a_{1}^{4} a_{2}^{6} b_{2}^{10} d_{2}^{6} e^{6} k^{8} \rho^{2}-24 a_{1}^{3} a_{2}^{6} b_{2}^{9} d_{2}^{5} e^{6} k^{9} \rho^{2} \\
& +4 a_{1}^{2} a_{2}^{6} b_{2}^{8} d_{2}^{4} e^{6} k^{10} \rho^{2} \text {. } \\
& \begin{aligned}
l_{b 2}= & a_{1}^{3} b_{2} d_{2}^{3}\left(-a_{1} b_{2} d_{2}+k\right)^{2}-2 a_{1}^{2} b_{2}^{2} d_{2}^{4}\left(d_{2}-a_{2} e\right) k \rho+4 a_{1} b_{2} d_{2}^{3} k^{2} \rho^{2} \\
& +2 e^{2}\left(-d_{2}+a_{2} e\right) k^{3} \rho^{3}, \\
l_{b 3}= & a_{1}^{2} d_{2}^{2}\left(-a_{1} b_{2} d_{2}+k\right)^{2}-8 a_{1} b_{2} d_{2}^{3}\left(d_{2}-a_{2} e\right) k \rho+4\left(-2 a_{2} d_{2} e^{3}\right. \\
& \left.+a_{2}^{2} e^{4}+d_{2}^{2}\left(1+e^{2}\right)\right) k^{2} \rho^{2} .
\end{aligned}
\end{aligned}
$$




\section{Acknowledgments}

The first author is grateful to CONACYT for financial support 10017-181247 given to this work. The second author would like to thanks the CONACYT for the grant 246073 for the sabbatical year at the Intituto de Matemáticas of the Universidad Nacional Autónoma de México and to the Departament de Matemàtiques of Universitat Autònoma de Barcelona for their hospitality, where part of this work was carried out. The third author is partially supported by a MINECO grant MTM2013-40998-P, an AGAUR grant number 2014SGR-568, and the grants FP7-PEOPLE-2012-IRSES 318999 and 316338.

\section{References}

1. C. A. Buzzi and J. Ilibre Hopf bifurcation in the full repressilator equations Math. Meth. Appl. Sci. (2015), 38 1428?1436.

2. V. Castellanos, J. Llibre and I. Quilantan. Simultaneous Periodic Orbits Bifurcating from Two Zero-Hopf Equilibria in a Tritrophic Food Chain Model J. of Applied Math and Phys. 1, 7 (2013), 1-38.

3. S.M. Baer, B. Li, and H.L. Smith, Multiple limit cycles in the standard model of three species competition for three essential resources, J. Math. Biol. 52 (2006), 745-760.

4. J.P. Francoise and J. Llibre, Analytical study of a higher-order hopf bifurcation in a tritrophic food chain model, Appl. Math. Comput. 217 (2011), 7146-7154.

5. C.S. Holling, Some characteristics of simple types of predation and parasitism, Canad. Entomol. 91 (1959), 385-398.

6. Yu. A. Kuznetsov, O. De Feo, and D. Rinaldi, Belyakov homoclinic bifurcations in a tritrophic food chain model, SIAM J. Appl. Math 62 (2001), 462-487.

7. Yu. A. Kuznetsov, Elements of applied bifurcation theory, third ed., Applied Mathematical Sciences, vol. 12, Springer-Verlag, New York, 2004.

8. R. May, Limit cycles in predator-prey communities, Science 177 (1972), 900-902. 